\title{
Annexe I
}

\section{FONDEMENTS CINÉTIQUE ET FLUIDE DE L'AÉRONOMIE}

\section{INTRODUCTION AUX PLASMAS}

\section{DÉFINITION}

La matière est généralement classée en trois états fondamentaux que sont le solide, le liquide et le gaz. Toutefois, lorsqu'un gaz est chauffé à des températures élevées, les atomes (ou molécules) qui le constituent sont soumis à des chocs très violents qui peuvent provoquer une ionisation de ce gaz. La température croissant, on peut aboutir à un gaz complètement ionisé, composé uniquement d'ions et d'électrons mais globalement neutre. Cet état est souvent qualifié de quatrième état de la matière. Il est celui qui prédominait aux instants initiaux de la création de l'univers (avant recombinaison ion-electron). Le physicien américain Irwin Langmuir a introduit en 1923 le terme de plasma pour caractériser cet état. Depuis, la terminologie plasma a été étendue et elle désigne un état dilué de la matière, analogue à un gaz, mais constitué de particules chargées, électrons et ions, en proportions telles que le milieu est globalement neutre.

Ainsi, si $n_{n}$ est la concentration de gaz neutre, $n_{i}$ celle de gaz ionisé et, le taux d'ionisation $\tau=\frac{n_{i}}{n_{n}+n_{i}}$ a une grande dynamique entre $O$ (gaz neutre non ionisé) et 1 (gaz complètement ionisé). Entre ces deux valeurs extrêmes, on trouve une multitude de plasmas dans divers milieux (figure $A I-1$ ).

Un gaz en équilibre thermodynamique a un taux d'ionisation dont la valeur est imposée par la loi de Saha, qui exprime que la concentration en espèces chargées résulte d'un équilibre thermodynamique entre mécanismes d'arrachement et de capture d'électrons. Cette loi montre que le taux d'ionisation croît sensiblement avec la température thermodynamique. Cependant, dans les plasmas des environnements planétaires, d'autres processus d'ionisation doivent être invoqués, car les températures sont trop faibles pour expliquer le taux d'ionisation mesuré ; aux altitudes ionosphériques ce taux d'ionisation varie entre $10^{-9}$ et 1 sur environ $1000 \mathrm{~km}$. Les rayonnements photoniques et les flux de particules de grandes énergies sont alors les causes principales de l'ionisation des constituants neutres. 


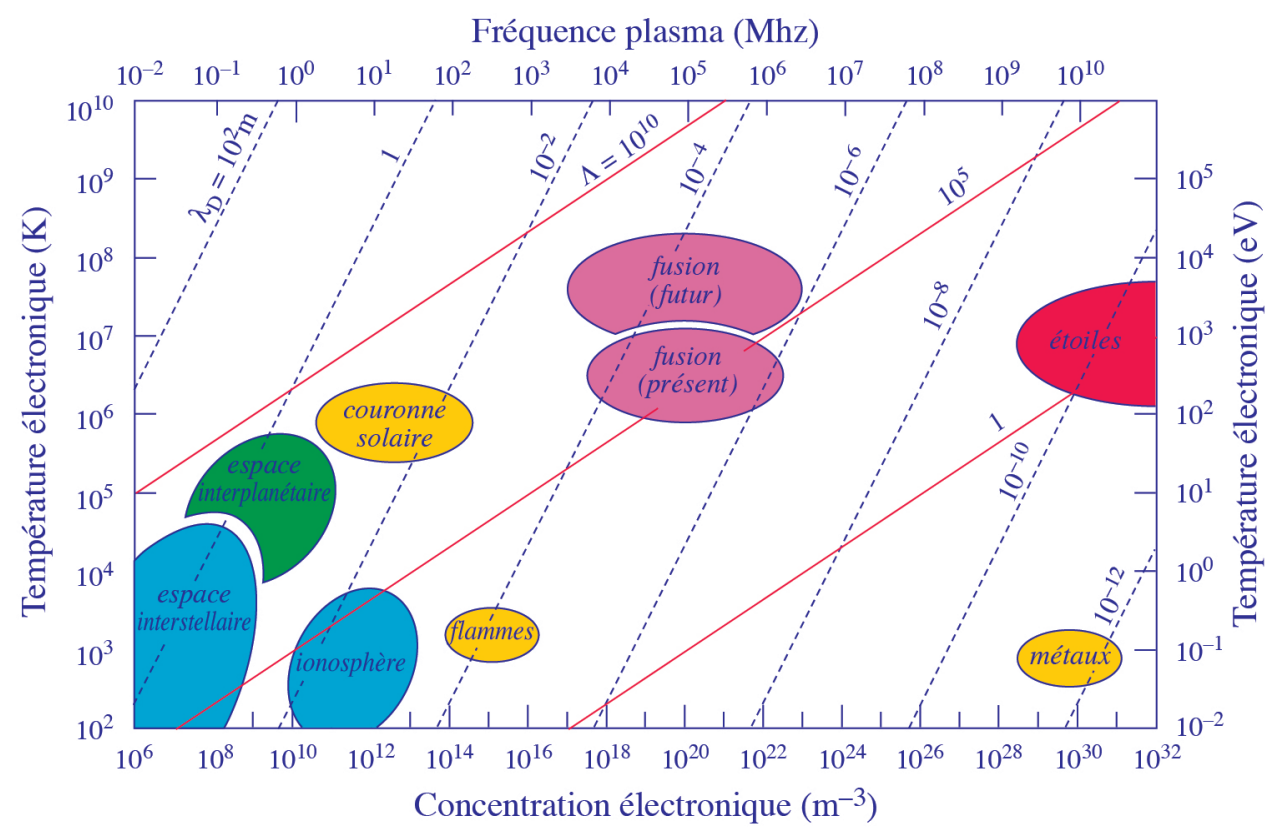

Figure AI-1 : domaine de densité (en abscisse) et de température (en ordonnée) pour différents types de plasmas

On a reporté les échelles de fréquence plasma et de longueur de Debye $\lambda_{D}$ définis dans l'annexe III, ainsi que celle du paramètre plasma $\Lambda$ qui caractérise la validité des hypothèses faites.

\section{INTERACTIONS PARTICULAIRES DANS UN PLASMA}

Le but n'est pas de décrire de manière exhaustive tous les mécanismes d'interaction que l'on peut rencontrer dans un plasma, mais plutôt de présenter la diversité des interactions entre particules en focalisant sur quelques uns. Les deux familles de populations (neutres et chargées) qui constituent le gaz sont sources de plusieurs types d'interactions. En nous restreignant aux gaz dilués en interactions élastiques, on peut essentiellement définir trois classes.

\section{a. Interaction entre particules neutres}

Plusieurs potentiels décrivant ce type d'interaction ont été proposés, le plus courant étant celui de Lennard-Jones dont une expression est donnée par :

$V(r)=4 \varepsilon\left(\left(\frac{\sigma}{r}\right)^{12}-\left(\frac{\sigma}{r}\right)^{\sigma}\right)$

où $\varepsilon$ est l'énergie des forces intermoléculaires et $\sigma$ représente la portée de ces forces. La figure A1-2 ci-dessous représente l'allure de ce potentiel en fonction de la distance $r$ séparant les particules en interactions (courbe en trait plein). 


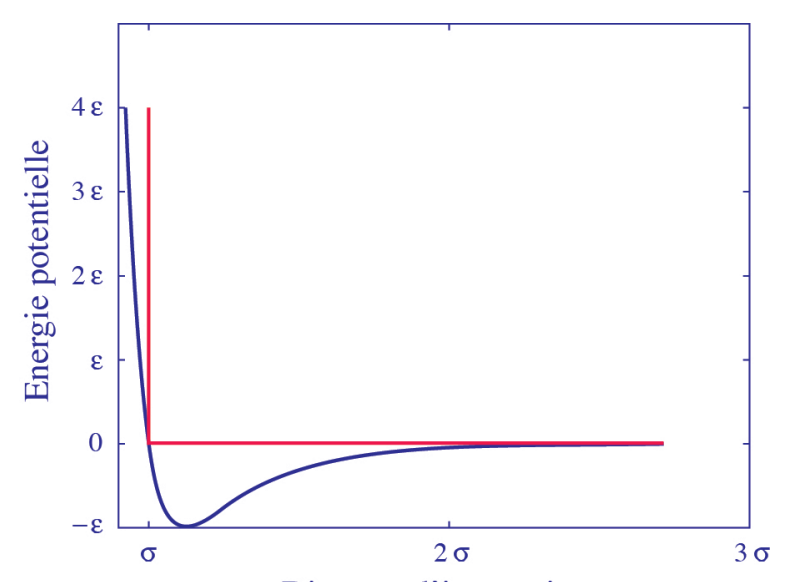

Distance d'interaction

\section{Figure A1-2 : potentiel d'interaction du type Lennard-Jones (bleu) et approximation par un modèle sphère dure (rouge)}

Dans cette expression, la composante en $r^{-6}$ caractérise les effets attractifs à grande distance (forces de Van der Waals) résultant de l'apparition de dipôles électrostatiques lors du rapprochement de particules neutres. La composante en $r^{-12}$ traduit, pour sa part, des comportements répulsifs quantiques beaucoup plus intenses à très courte distance.

On constate que la portée de l'interaction est très faible. En effet, au delà d'une distance de l'ordre de $2 \sigma$, le potentiel est quasiment nul, et son gradient extrêmement faible. La dépendance principale en $r^{-6}$ donne à l'énergie d'interaction d'une particule avec une sphère de gaz de rayon $R$, centrée sur la particule, une dépendance en $R^{-3}$, qui décroît rapidement vers 0 lorsque $R$ croît. La sphère d'influence de la particule est donc restreint à l'environnement très proche de la particule (quelques $\sigma$ ) : nous avons affaire à une interaction à très courte portée. On peut alors avantageusement simplifier cette interaction en utilisant un modèle "boule de billard", ou sphère dure (courbe en rouge sur la figure A1-2), défini par :

$$
\left\{\begin{array}{l}
V(r)=\infty \text { pour } r \leq \sigma \\
V(r)=0 \text { pour } r>\sigma
\end{array}\right.
$$

où $\sigma$ représente alors la portée de l'interaction.

Elle caractérise également la distance minimale d'approche : dans un modèle classique de boules de billard, $\sigma$ représente la somme des diamètres des boules qui entrent en collision. Cette portée est inférieure à la distance interparticulaire moyenne entre particules neutres du gaz.

Le mécanisme des boules de billard autorise une approche commode des interactions, et notamment il permet de développer des expressions simples des fréquences de collisions. 


\section{b. Interactions entre particules neutres et particules chargées}

La deuxième classe correspond aux interactions entre particules neutres et particules chargées. Lorsqu'une particule chargée s'approche d'une particule neutre, il y a induction d'un dipôle électrostatique au niveau de cette dernière et une force d'attraction apparaît. Le potentiel dont dérive cette force prend la forme suivante :

$V(r)=-\frac{\alpha Z e^{2}}{4 \pi \varepsilon_{0} r^{4}}$

où $\alpha$ est la polarisabilité de la molécule neutre et $Z$ est le nombre de charge de l'ion. Ce type d'interaction est également à courte portée : l'énergie d'interaction d'une particule avec une sphère de gaz de rayon $R$, centrée sur la particule, a une dépendance en $R^{-1}$.

\section{c. Interactions entre particules chargées}

La troisième classe concerne les interactions entre particules chargées. Deux particules de charge respectives $Z_{1} e$ et $Z_{2} e$, distantes de $r$, ont un potentiel d'interaction coulombien de la forme :

$V(r)=\frac{Z_{1} Z_{2} e^{2}}{4 \pi \varepsilon_{\mathrm{o}} r}$

Contrairement aux cas précédents, ce type d'interaction est à très longue portée. En effet, l'énergie d'interaction d'une particule chargée avec une sphère de particules chargées de rayon $\mathrm{R}$ centrée sur la particule, a une dépendance en $R^{2}$ : l'énergie diverge très vite avec la distance à la particule. Cette apparente aberration est liée au caractère collectif de l'interaction, qui n'est pas inclus dans ce calcul. Les électrons ont tendance à s'agglomérer autour des ions, plus lourds, de manière à compenser le potentiel coulombien créé par l'ion. De fait, au delà d'une distance $\lambda_{D}$, appelée longueur de Debye, le potentiel de l'ion est quasiment compensé par le potentiel des électrons ambiants : il y a écrantage du potentiel. La longueur $\lambda_{D}$ correspond approximativement à la distance à l'ion pour laquelle l'énergie d'attraction induite par cet ion est égale à l'énergie volumique d'agitation thermique des électrons du plasma. On peut considérer qu'à des distances d'observation supérieures à $\lambda_{D}$, le milieu est neutre. Le calcul rigoureux de cette distance est proposé dans l'annexe III. Suivant les milieux observés (voir figure $A I-1$ ), la longueur de Debye peut prendre des valeurs très grandes $\left(\approx 10^{8} \mathrm{~m}\right.$ dans les espaces interplanétaires et interstellaires, ou la couronne solaire) ou modérées $(<1 \mathrm{~m}$ dans les étoiles et les ionosphères planétaires). Cette grandeur caractérise de fait la portée de l'interaction entre particules chargées. Celle-ci, dans les plasmas qui nous intéressent, est toujours très largement supérieure à la distance interparticulaire moyenne entre particules chargées du gaz; cela traduit le comportement collectif du gaz de particules chargées (voir annexe $I I I$ ).

Dans la suite de cette annexe, nous allons introduire la manière dont on décrit le plasma en tenant compte de ces interactions interparticulaires. 


\section{APPROCHE STATISTIQUE}

\section{FONCTION DE DISTRIBUTION}

Dans le cas d'une population constituée d'un grand nombre de particules, l'approche particulaire, qui consiste à suivre chaque particule individuellement, n'est plus possible. Il faut alors choisir une approche statistique où on n'étudiera plus que quelques propriétés de cette population. En premier lieu, il n'est plus envisageable de repérer chaque particule et de fait elles deviennent toutes équivalentes : c'est le principe d'indiscernabilité. Cette approche statistique consiste à caractériser la population par un certain nombre d'états accessibles aux particules et la probabilité de répartition de la population sur les différents états. Comme le nombre de particules est connu, plutôt que par sa probabilité de répartition, on préférera caractériser le nombre de particules sur chaque état admissible. Si on note $\Omega$ l'espace de ces états admissibles, on introduit alors une fonction $f$, définie sur $\Omega$ et appelée fonction de distribution, telle que pour $\vec{X} \in \Omega, f(\vec{X}) d \tau$ représente le nombre de particules dans le voisinage $d \tau$ de $\vec{X}$ ( $d \tau$ s'apparente à un volume élémentaire dans $\Omega$ ).

Dans les gaz qui nous intéressent (gaz atomiques principalement), les états qui sont importants à caractériser sont d'une part la position $\vec{r}$ et d'autre part l'impulsion $\vec{p}$ (ou quantité de mouvement) ou la vitesse $\vec{v}$, car pour des systèmes à forces conservatives, les actions qui s'exercent sur les particules du gaz ne dépendent que de ces grandeurs et leur connaissance permet d'accéder à l'évolution du gaz (principe de Poincaré). Dans certains cas, la connaissance de la position s'avère être secondaire et l'état à caractériser sera le module de la vitesse ou de l'impulsion ou encore, ce qui est équivalent, la valeur de l'énergie totale (voir paragraphe suivant).

L'espace des position - impulsion (ou position - vitesse) est appelé espace des phases (espace à 6 dimensions) et la fonction de distribution $f_{p}(\vec{r}, \vec{p}, t)$ que l'on bâtit sur cet espace est telle que $f_{p}(\vec{r}, \vec{p}, t) d^{3} \vec{r} d^{3} \vec{p}$ représente le nombre de particules qui, à l'instant $t$, sont présentes dans le volume $d^{3} \vec{r}$ centré sur $\vec{r}$ et qui ont l'impulsion $\vec{p}$ à $d^{3} \vec{p}$ près.

La dimension de $f_{p}$ est donc $\mathrm{L}^{-6} \mathrm{~T}^{3} \mathrm{M}^{3}$ (dans une représentation position-vitesse la dimension de la fonction de distribution serait $\mathrm{L}^{-6} \mathrm{~T}^{3}$ ). Elle représente une photographie du système à chaque instant: ainsi, $\{\vec{r}, \vec{p}\}$ constitue-t-il une base de l'espace des phases. En d'autres termes, $\vec{r}$ n'est fonction ni de $t$, ni de $\vec{p}$, et $\vec{p}$ n'est fonction ni de $t$ ni de $\vec{r}$. L'homogénéité, ou uniformité spatiale, est le caractère d'invariance par translation spatiale : dans le cas homogène, $f_{p}$ ne dépend pas de $\vec{r}$. L'isotropie est l'homogénéité de l'espace des vitesses. Dans le cas isotrope, $f_{p}$ ne dépend pas de $\vec{p}$. Enfin, la stationnarité est définie par une fonction de distribution ne dépendant pas explicitement du temps. Par ailleurs, la fonction de distribution telle qu'elle est définie ici n'est pas normalisée ainsi qu'on la trouve 
parfois en statistique. Son intégrale sur l'espace des phases est égale au nombre de particules constituant la population.

Une telle représentation, avec une fonction de distribution dite à une espèce, n'est pas apte, a priori, à prendre en compte les interactions qui existent au sein du gaz car toutes les particules étant équivalentes, aucun couplage n'est possible. Toutefois, dans les gaz dilués, comme ceux que nous rencontrons en aéronomie, cette représentation est suffisante ; la caractérisation des interactions sera faite en adjoignant un opérateur de collisions à l'équation de transport de la fonction de distribution. La construction de cet opérateur s'appuie sur le caractère dilué du gaz qui entraîne que les particules sont faiblement corrélées.

\section{FONCTION DE DISTRIBUTION DE MAXWELL}

La fonction de distribution joue un rôle important dans l'étude de la dynamique des systèmes. Nous allons donc déterminer la forme qu'elle prend dans le cas d'un gaz à l'équilibre, dont les particules interagissent suivant un modèle de type sphère dure (portée d'interaction nulle) décrit plus loin dans ce chapitre.

Nous considérerons un gaz au repos (vitesse moyenne de déplacement nulle) contenant $N$ particules. A l'échelle macroscopique, en plus du nombre de particules, nous caractériserons le gaz par son énergie totale $E$, constante. L'hypothèse d'une interaction de type sphère dure traduit alors la propriété que les différentes interactions ont pour principal effet de redistribuer l'énergie entre les particules ou, ce qui revient au même, de redistribuer les particules sur les différents niveaux d'énergie accessibles.

\section{a. Niveaux d'énergie quantifiés}

Nous supposerons d'abord que les particules ne peuvent prendre que des valeurs discrètes de l'énergie. On définira les niveaux d'énergie quantifiés $\left(E_{j}\right)_{j \in J}$ (où $J$ est un ensemble discret) accessibles aux particules. A l'équilibre, on notera $N_{j}$ le nombre de particules parmi les $N$ qui ont l'énergie $E_{j}$. Le système n'étant pas figé, les particules changent de niveaux d'énergie au cours du temps, mais le nombre de particules par niveau reste constant : c'est la manifestation de l'indiscernabilité. On a alors les relations :

$$
\left\{\begin{array}{l}
N=\sum_{j \in J} N_{j} \\
E=\sum_{j \in J} N_{j} E_{j}
\end{array}\right.
$$

On définit le nombre de complexion qui est le nombre de configurations possibles pour le gaz :

$$
W=\frac{N !}{\prod_{j \in J} N_{j} !}
$$


$W$ correspond au nombre de possibilités de répartir les $N$ particules sur les différents niveaux d'énergie. Si $\operatorname{Card}(J)$ est supérieur à $N$, alors certains niveaux ne sont pas remplis, puisque les $N_{j}$ sont des entiers.

On peut raisonnablement supposer que la configuration la plus probable pour le système est celle correspondant à l'état le plus désordonné possible, sous les contraintes de la quantité d'information connue sur le système, soit les relations $A I-5$.

Pour des raisons d'extensivité, on est amené à introduire l'entropie, la fonction mesurant le désordre, sous la forme :

$S=k \log (W)$

En prenant $k=k_{b}=1,3810^{-23} \mathrm{~J} \mathrm{~K}^{-1}$, on obtient des grandeurs thermodynamiques mesurées dans le système SI (notamment la température est donnée en Kelvin), et en prenant $k=8,62510^{-5} \mathrm{eV} \mathrm{K}^{-1}$, on obtient des grandeurs thermodynamiques mesurées dans le système CeVs (voir annexe $I I$ ). Pour $N$ grand, en utilisant la formule de Stirling, on peut développer l'entropie sous la forme :

$S \cong k_{b} \sum_{j \in J} N_{j} \log N_{j}+S_{o}$

AI-8

où $S_{o}$ est une constante. La situation la plus désordonnée correspond à un extrémum de l'entropie. En considérant les contraintes $A I-5$ dans le mécanisme d'extrémalisation, nous trouvons comme répartition suivante :

$N_{j}=\frac{1}{Z} \mathrm{e}^{-\frac{E_{j}}{k_{b} T}}$

où $Z$ est un coefficient de normalisation et $\frac{1}{T}$ est une grandeur (le multiplicateur de Lagrange associé à l'énergie) introduite pour résoudre le problème d'extrémalité. $T$ possède toutes les propriétés d'une température thermodynamique et correspond effectivement à la température du système, si l'on a pris comme constante de normalisation $k_{b}$.

$Z$ et $T$ sont définis par les relations $A I-5$, soit :

$$
\left\{\begin{array}{l}
N=\frac{1}{Z} \sum_{j \in J} \mathrm{e}^{-\frac{E_{j}}{k_{b} T}} \\
E=\frac{1}{Z} \sum_{j \in J} \mathrm{e}^{-\frac{E_{j}}{k_{b} T} E_{j}}
\end{array}\right.
$$

Le facteur e $\mathrm{e}^{-\frac{E_{j}}{k_{b} T}}$ est appelé facteur de Boltzmann. Dans cette solution, on constate que tous les niveaux d'énergie sont occupés et que les $N_{j}$ sont réels; ceci est une conséquence du procédé d'extrémalisation qui consiste à dire que les fonctions sont continûment dérivables pour utiliser la propriété du gradient d'être nul en un extrémum. 


\section{b. Niveaux d'énergie continus}

Dans la réalité, les niveaux accessibles forment des intervalles continus. En supposant qu'il n'y a qu'un seul intervalle $\left[0 ; E_{m a x}\right]$, on peut reprendre l'étude précédente en écrivant :

$E_{j}=\left(j+\frac{1}{2}\right) d E \quad \forall j \in[0 ; p-1] \quad$ avec $\quad p d E=E_{\max }$

et en attribuant l'énergie $E_{j}$ à toute particule dont l'énergie se trouve dans l'intervalle $\left[E_{j}-\frac{d E}{2} ; E_{j}+\frac{d E}{2}[\right.$.

On choisit de noter $d N_{j}$ le nombre de particules sur le niveau d'énergie $E_{j}$. Si la largeur $d E$ tend vers 0 , alors le nombre de particules $d N_{j}$ tend également vers 0 , mais le rapport $\frac{d N_{j}}{d E}$ tend vers une limite finie.

En effet, en reprenant $A I-10$, nous pouvons écrire :

$N=\sum_{j=0}^{p-1} \frac{1}{Z} \mathrm{e}^{-\frac{d E}{2 k_{b} T}} \mathrm{e}^{-\frac{j d E}{k_{b} T}}=\frac{1}{Z} \mathrm{e}^{-\frac{d E}{2 k_{b} T}} \frac{1-\mathrm{e}^{-\frac{E_{\text {max }}}{k_{b} T}}}{1-\mathrm{e}^{-\frac{d E}{k_{b} T}}}$

Si l'axe réel est entièrement accessible $\left(E_{\max } \rightarrow \infty\right)$, on a :

$N=\frac{1}{Z} \frac{\mathrm{e}^{-\frac{d E}{2 k_{b} T}}}{1-\mathrm{e}^{-\frac{d E}{k_{b} T}}}$

ou :

$\frac{1}{Z}=N \mathrm{e}^{\frac{d E}{2 k_{b} T}}\left(1-\mathrm{e}^{-\frac{d E}{k_{b} T}}\right)$

En faisant tendre $d E$ vers 0 , on peut alors développer $A I-14$ sous la forme :

$\frac{1}{Z}=\frac{N}{k_{b} T} d E$

et donc :

$d N_{j}=\frac{N}{k_{b} T} \mathrm{e}^{-\frac{E_{j}}{k_{b} T}} d E$

soit un rapport qui admet une limite finie. On introduit alors la fonction de distribution du gaz en énergie $f_{e}$ en posant :

$f_{e}(E)=\frac{N}{k_{b} T} \mathrm{e}^{-\frac{E}{k_{b} T}}$

qui vérifie : 


$$
\left\{\begin{array}{l}
N=\int_{0}^{\infty} f_{e}(\varepsilon) d \varepsilon \\
E=\int_{0}^{\infty} f_{e}(\varepsilon) \varepsilon d \varepsilon
\end{array}\right.
$$

\section{c. Distribution de Maxwell}

Pour une particule donnée de masse $m$, son énergie est constituée par son énergie cinétique $K$ et son énergie potentielle $U$, si l'on suppose que toutes les forces agissant sur la particule sont conservatives et donc dérivent d'un potentiel. Nous avons supposé que toute l'énergie cinétique était de l'énergie de translation et à l'exclusion de toute autre contribution, du type énergie cinétique de rotation ou de vibration. Ceci suppose implicitement que nous considérons des particules atomiques, choix que nous maintiendrons dans la suite de ce chapitre.

Or $K$ n'est fonction que de l'impulsion $\vec{p}$ et $U$ n'est fonction que de la position $\vec{r}$ (puisque les forces sont conservatives). Nous pouvons donc exprimer différemment la fonction de distribution en énergie et définir une fonction de distribution simple en position et impulsion $f_{p}(\vec{r}, \vec{p})$ proportionnelle à $\frac{N}{k_{b} T} \mathrm{e}^{-\frac{p^{2}}{2 m k_{b} T}} \mathrm{e}^{-\frac{U(r)}{k_{b} T}}$ (on notera $\beta$ le coefficient de proportionnalité).

Si on note $n(\vec{r})$ le concentration volumique de particules présentes à la position $\vec{r}$, nous pouvons écrire :

$$
n(\vec{r})=\iiint_{f_{p}}(\vec{r}, \vec{p}) d^{3} \vec{p}
$$

et donc :

$n(\vec{r})=\frac{\beta N}{k_{b} T} \mathrm{e}^{-\frac{U(r)}{k_{b} T} \iiint \mathrm{e}^{-\frac{p^{2}}{2 m k_{b} T}} d^{3} \vec{p}}$

Comme $\int_{-\infty}^{+\infty} \mathrm{e}^{-a u^{2}} d u=\sqrt{\frac{\pi}{a}}$, nous avons $\iiint_{\mathrm{e}^{-}}^{-\frac{p^{2}}{2 m k_{b} T}} d^{3} \vec{p}=\left(2 \pi m k_{b} T\right)^{3 / 2}$. On trouve :

$f_{p}(\vec{r}, \vec{p})=n(\vec{r}) \frac{1}{\left(2 \pi m k_{b} T\right)^{3 / 2}} \mathrm{e}^{-\frac{p^{2}}{2 m k_{b} T}}$

Si les particules ne sont pas soumises à un champ électromagnétique et qu'elles ne sont pas relativistes, on peut écrire $\vec{p}=m \vec{v}$ et $d^{3} \vec{p}=m^{3} d^{3} \vec{v}$.

On définit alors la fonction de distribution de Maxwell $f(\vec{r}, \vec{v})$ par : 
$f(\vec{r}, \vec{v})=n(\vec{r})\left(\frac{m}{2 \pi k_{b} T}\right)^{3 / 2} \mathrm{e}^{-\frac{m v^{2}}{2 k_{b} T}}$

Cette fonction caractérise la population dans une représentation position-vitesse. Elle montre que localement, dans une unité de volume élémentaire, la distribution de vitesse ne se fait qu'en vitesse. $n(\vec{r})$ contient toute l'information sur la répartition des particules en position, induite par l'action des forces extérieures. Ainsi on caractérise chaque population du système par une fonction de distribution propre.

Nous verrons plus loin l'importance que revêt cette fonction de distribution pour l'étude de la dynamique des gaz.

\section{LES MOMENTS DE LA FONCTION DE DISTRIBUTION}

La description fine offerte par la fonction de distribution n'est pas toujours appropriée, surtout si on étudie le système à une échelle macroscopique. On caractérise alors le gaz par des paramètres correspondant à des moyennes sur l'espace des vitesses de fonctions polynomiales du vecteur vitesse (que nous définissons par la suite) pondérées par la fonction de distribution ; ce sont les moments de la fonction de distribution.

Nous considérerons dans la suite une fonction de distribution $f(\vec{r}, \vec{v}, t)$ dépendant a priori du temps $t$.

\section{MOMENT SCALAIRE D'ORDRE 0 : CONCENTRATION DU GAZ}

Le moment d'ordre 0 est associé polynôme 1 et vaut donc :

$n(\vec{r}, t)=\iiint_{1} f(\vec{r}, \vec{v}, t) d^{3} \vec{v}$

Comme nous l'avons vu précédemment, ceci définit la concentration volumique du gaz à la position $\vec{r}$. Si le gaz est constitué de particules de masse $m$, nous pouvons encore définir la masse volumique :

$\rho(\vec{r}, t)=m n(\vec{r}, t)$

Si les particules ont une charge $q$, nous définirons la charge volumique :

$\rho_{c}(\vec{r}, t)=q n(\vec{r}, t)$

La charge volumique globale est la somme des charges volumiques de toutes les espèces. Dans l'hypothèse classique de neutralité du plasma, elle vaut zéro. En règle générale, nous mentionnerons à l'introduction d'un paramètre, ses dépendances vis à vis des grandeurs spatiales et temporelle, mais nous les omettrons dans les utilisations ultérieures afin de ne pas alourdir les notations. 


\section{MOMENT VECTORIEL D'ORDRE 1 : FLUX DE PARTICULES ET VITESSE MOYENNE D'ÉCOULEMENT}

Le moment vectoriel d'ordre 1 est associé au polynôme $\vec{v}$ et il est défini de la manière suivante :

$\vec{\Phi}(\vec{r}, t)=\iiint \vec{v} f(\vec{r}, \vec{v}, t) d^{3} \vec{v}$

$\vec{\Phi}(\vec{r}, t)$ est le flux de particules qui s'écoulent par unité de temps et de surface. On définit alors la vitesse moyenne de l'écoulement $\vec{u}(\vec{r}, t)$ par:

$\vec{u}(\vec{r}, t)=\frac{1}{n} \vec{\Phi}=\frac{1}{n} \iiint \vec{v} f(\vec{r}, \vec{v}, t) d^{3} \vec{v}$

Comme cette vitesse revêt une grande importance dans les équations de transport, on considère souvent qu'elle est le moment vectoriel d'ordre 1 de la fonction de distribution. La quantité de mouvement $\vec{\pi}(\vec{r}, t)$ de la population est définie par :

$\vec{\pi}(\vec{r}, t)=m n \vec{u}=m \vec{\Phi}$

Si les particules ont une charge $q$, nous définirons la densité de courant $\vec{j}(\vec{r}, t)$ :

$\vec{j}(\vec{r}, t)=q n \vec{u}=q \vec{\Phi}$

La densité totale de courant est la somme des densités de courants des différentes espèces. La définition AI-29 montre que cette densité de courant est positive dans le sens des ions positifs. A la position $\vec{r}$, nous pouvons nous placer dans le référentiel local qui se déplace à la vitesse $\vec{u}$ avec le gaz (référentiel inertiel). Une particule de vitesse $\vec{v}$ dans le référentiel de départ (référentiel du laboratoire) a ainsi une vitesse $\vec{c}$, souvent appelée vitesse thermique ou vitesse "particulière" (traduction de l'anglais peculiar) dans le référentiel local, telle que :

$\vec{v}=\vec{u}+\vec{c}$

Nous pouvons alors définir une fonction de distribution $f_{c}(\vec{r}, \vec{c}, t)$ dans le référentiel local, en écrivant que le nombre de particules dans un élément de volume de l'espace des phases est conservé lors du changement de référentiel, soit :

$f(\vec{r}, \vec{v}, t) d^{3} \vec{v}=f_{c}(\vec{r}, \vec{c}, t) d^{3} \vec{c}$

Comme nous avons $d^{3} \vec{v}=d^{3} \vec{c}$, nous en déduisons donc :

$f_{c}(\vec{r}, \vec{c}, t)=f(\vec{r}, \vec{u}+\vec{c}, t)$ 
Cette nouvelle fonction de distribution vérifie les propriétés suivantes :

$n=\iiint_{f_{c}}(\vec{r}, \vec{c}, t) d^{3} \vec{c}$

et, par définition :

$\iiint \vec{c} f_{c}(\vec{r}, \vec{c}, t) d^{3} \vec{c}=\overrightarrow{0}$ AI-34

On considérera souvent que dans le référentiel local, les particules sont en équilibre thermodynamique et que leur fonction de distribution s'apparente à une fonction de distribution de Maxwell, caractérisée par une température $T(\vec{r}, t)$, dépendant du temps $t$ et de la position $\vec{r}$. Dans ce cas, la fonction de distribution locale $f_{c}$ s'écrit :

$f_{c}(\vec{r}, \vec{c})=n\left(\frac{m}{2 \pi k_{b} T}\right)^{3 / 2} \mathrm{e}^{-\frac{m c^{2}}{2 k_{b} T}}$

qui justifie la dénomination de "vitesse thermique" pour le vecteur $\vec{c}$. En effet, dans le référentiel local, les particules ne sont mues que par l'agitation thermique, et les collisions à l'intérieur du gaz redistribuent l'énergie totale $\frac{3}{2} n k_{b} T$ entre les particules; $\vec{c}$ est alors le vecteur qui caractérise cette redistribution d'énergie.

\section{MOMENT SCALAIRE D'ORDRE 2 : ÉNERGIE TOTALE ET THERMIQUE}

Le moment scalaire d'ordre 2 est associé au polynôme $\frac{1}{2} \boldsymbol{m} \boldsymbol{v}^{2}$ et correspond à l'énergie totale $\varepsilon$ des particules placées en $\vec{r}$ :

$\varepsilon(\vec{r}, t)=\iiint \frac{1}{2} \boldsymbol{m} v^{2} f(\vec{r}, \vec{v}, t) d^{3} \vec{v}$

Or, nous avons $v^{2}=u^{2}+c^{2}+2 \vec{u} \cdot \vec{c}$ et l'énergie s'exprime donc sous la forme :

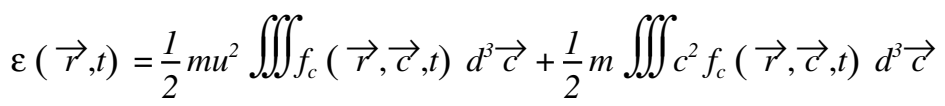

$$
\begin{aligned}
& +m \vec{u} \cdot \iiint \vec{c} f_{c}(\vec{r}, \vec{c}, t) d^{3} \vec{c}
\end{aligned}
$$

Soit, d'après les relations $A I-33$ et $A I-34$ :

$\varepsilon(\vec{r}, t)=\frac{1}{2} n m u^{2}+\frac{1}{2} m \iiint c^{2} f_{c}(\vec{r}, \vec{c}, t) d^{3} \vec{c}$ 
La relation $A I-38$ est une caractérisation du théorème de Koenig, qui énonce que l'énergie d'un système peut se décomposer en l'énergie cinétique du centre de masse (affecté de la masse du système et de la vitesse de déplacement du système) et de l'énergie cinétique du système dans le référentiel barycentrique.

Ici, le référentiel barycentrique est le référentiel local et $\frac{1}{2} n m u^{2}$ eprésente l'énergie cinétique volumique macroscopique du gaz qui s'écoule à la vitesse $\vec{u}$.

$\varepsilon_{t h}(\vec{r}, t)=\frac{1}{2} m \iiint \int_{c}^{2} f_{c}(\vec{r}, \vec{c}, t) \quad d^{3} \vec{c}$ est l'énergie cinétique dans le référentiel barycentrique, qui correspond à l'énergie d'agitation dans ce référentiel ; on l'appelle donc énergie thermique (ou énergie d'agitation thermique) du gaz.

Si $f_{c}$ est la fonction de distribution de Maxwell donnée en $A I-35$, on trouve :

$\varepsilon_{t h}=\frac{3}{2} n k_{b} T$

On introduit alors la pression cinétique du gaz $p(\vec{r}, t)$ :

$p(\vec{r}, t)=\frac{1}{3} m \iiint_{c^{2}} f_{c}(\vec{r}, \vec{c}, t) d^{3} \vec{c}$

qui nous permet d'écrire :

$\varepsilon_{t h}=\frac{3}{2} p$

Cette équation caractérise une loi d'état du type gaz parfait :

$p=n k_{b} T$

et relie la température cinétique du gaz $T$ à sa pression cinétique.

Dans les cas des gaz dilués, où les interactions sont faibles, la pression cinétique est très largement supérieure à la pression intermoléculaire résultant des interactions entre particules et par conséquent, la pression du gaz est essentiellement la pression cinétique. L'équation précédente constitue alors une généralisation de la loi des gaz parfaits.

Si nous avons un mélange de plusieurs espèces, $p_{s}$ représente la pression partielle de l'espèce $s$, calculée comme précédemment, et la pression totale est donnée par la somme des pressions partielles.

L'information importante que l'on peut déduire de la fonction de distribution est celle concernant l'agitation thermique : nous venons de quantifier macroscopiquement cette agitation par une pression ou une température. Toutefois, on peut extraire d'autres informations macroscopiques sur l'énergie. 


\section{MOMENT TENSORIEL D'ORDRE 2 : TENSEUR DE PRESSION ET DE CONTRAINTE}

\section{a. Tenseurs}

Une différence fondamentale entre un corps et un espace vectoriel est la présence d'une loi de composition interne multiplicative dans le corps alors que la loi de composition multiplicative est externe dans le cas d'un espace vectoriel. Or en physique, il est parfois nécessaire de "multiplier" entre eux des vecteurs. Pour cela on a donc généralisé aux vecteurs la notion de multiplication de scalaires en introduisant la notion de tenseur. Nous ne développerons pas la théorie derrière l'objet mathématique, et nous nous contenterons de présenter les tenseurs utilisés en physique.

Définition : $E$ désignant un espace vectoriel de dimension $n$, on appelle tenseur d'ordre $p$ toute forme $p$-linéaire définie sur $E$.

Comme souvent en mathématique, on confond la fonction avec sa représentation. Les tenseurs n'échappent pas à cette règle.

Un tenseur d'ordre 1 est une forme linéaire. Or on identifie classiquement une forme linéaire à un vecteur (la forme linéaire étant alors le produit scalaire avec ce vecteur). Par extension, on associera un tenseur d'ordre 1 à un vecteur.

Un tenseur d'ordre 2 est une forme bilinéaire, comme l'est le produit scalaire. Or dans une base donnée de l'espace vectoriel $E$, une forme bilinéaire admet une représentation matricielle et par extension nous identifierons un tenseur d'ordre 2 à une matrice, dans une base donnée.

Définition : étant donné deux tenseurs $T_{1}$ et $T_{2}$ définis sur $E$, d'ordre respectifs $p$ et $q$, on définit le produit tensoriel de $T_{1}$ par $T_{2}$, noté $T_{1} \otimes T_{2}$, de la manière suivante :

$$
\begin{aligned}
T_{1} \otimes T_{2}: \quad & E^{p} \times E^{q} \longrightarrow \Re \\
& (\vec{u}, \vec{v}) \longrightarrow T_{l}(\vec{u}) T_{2}(\vec{v})
\end{aligned}
$$

où $\Re$ est le corps des réels. Le tenseur $T_{1} \otimes T_{2}$ est d'ordre $p+q$.

Ainsi, si on considère deux vecteurs $\vec{u}$ et $\vec{v}$ (qui sont également des tenseurs d'ordre 1 ), leur produit tensoriel sera défini par :

$$
\begin{array}{ll}
\vec{u} \otimes \vec{v}: \quad & E^{p} \times E^{q} \longrightarrow \Re \\
& (\vec{x}, \vec{y}) \longrightarrow(\vec{u} \cdot \vec{x})(\vec{v} \cdot \vec{y})
\end{array}
$$

où $(\vec{u} \cdot \vec{x})$ exprime le produit scalaire des vecteurs $\vec{u}$ et $\vec{x}$. Ce produit tensoriel est un tenseur d'ordre 2. Si on considère une base de $E$ sur laquelle $\vec{u}$ a pour composantes $\left(u_{i}\right)_{i=1 . . n}$ et $\vec{v}$ a pour composantes $\left(v_{j}\right)_{j=1 . . n}$, alors le produit tensoriel $\vec{u} \otimes \vec{v}$ admet comme représentation matricielle la matrice $T$ de composantes : $T_{\mathrm{ij}}=u_{i} v_{j}$. 
En généralisant, si $T_{1}$ est un tenseur d'ordre $p$ de représentation $\left[\left(T_{1}\right)_{i_{1} \ldots i_{p}}\right]_{i_{1}, \ldots, i_{p}=1 . . n}$ et $T_{2}$ un tenseur d'ordre $q$ de représentation $\left[\left(T_{2}\right)_{j_{1} \ldots j_{q}}\right]_{j_{1}, \ldots, j_{q}=1 . . n}$, le tenseur $T_{1} \otimes T_{2}$ a pour représentation : $\left[\left(T_{1}\right)_{i_{1} \ldots i_{p}}\left(T_{2}\right)_{j_{1} \ldots j_{q}}\right]_{i_{1}, \ldots, i_{p} j_{1}, \ldots, j_{q}=1 . . n}$.

Définition : on appelle contraction d'un tenseur l'opération qui consiste, après avoir choisi deux indices, à égaler et à sommer par rapport à cet indice deux fois répété.

La contraction de deux indices d'un tenseur d'ordre $p$ est alors un tenseur d'ordre $p-2$. La contraction peut être définie sur n'importe quels indices dès lors que l'un correspond à un indice sur $E^{p}$ et l'autre à un indice sur $E^{q}$.

Par exemple le tenseur $T$ d'ordre 5 de représentation $\left(T_{i_{1} i_{i} i_{3}, j_{2}}\right)_{i_{1}, i_{2}, i_{3} j_{1} j_{2}=1 . . n}$ contracté sur les indices 2 et 5 est le tenseur $T^{\prime}$ de représentation : $\left(\sum_{k=1}^{n} T_{i_{1} k i_{2} j_{l} k}\right)_{i, i_{2}, j_{l}=1 . . n}$.

Définition : étant donné deux tenseurs $T_{1}$ et $T_{2}$, on définit la multiplication contractée, notée $T_{1}: T_{2}$, le tenseur $T$ obtenu par la double contraction du produit tensoriel $T_{l} \otimes T_{2}$ sur l'avant dernier indice de $T_{1}$ et le premier indice de $T_{2}$ puis sur le dernier indice de $T_{1}$ et le second indice de $T_{2}$. Si $T_{1}$ est un tenseur d'ordre $p$ et $T_{2}$ est un tenseur d'ordre $q, T_{1}: T_{2}$ est un tenseur d'ordre $p+q-4$.

Ainsi, si $T_{1}$ est un tenseur d'ordre 3 de représentation $\left[\left(T_{1}\right)_{i_{1} i_{2} i_{3}}\right]_{i_{1}, i_{2}, i_{3}=1 . . n}$ et $T_{2}$ un tenseur d'ordre 2 de représentation $\left[\left(T_{2}\right)_{j_{j} j_{2}}\right]_{j_{l}, j_{2}=1 . . n}, T_{1}: T_{2}$ est un tenseur d'ordre 1 (soit un vecteur) qui a pour représentation : $\left(\sum_{k, l=1}^{n}\left(T_{1}\right)_{i k l}\left(T_{2}\right)_{k l}\right)=1 . . n$

Si un tenseur $T$ est dépendant d'un point de l'espace $\vec{x}$ de composantes $\left(x_{i}\right)_{i=1 . . n}$ dans une base donnée, on peut définir certaines opérations sur ce tenseur :

- en particulier, on peut définir le gradient du tenseur, noté $\vec{\nabla} T$, qui est un tenseur d'un ordre supérieur d'une unité à l'ordre du tenseur $T$;

Pour un tenseur d'ordre $1, \vec{\nabla} T$ est le tenseur d'ordre 2 qui dans une base donnée admet comme représentation la matrice $:\left(\frac{\partial T_{i}}{\partial x_{j}}\right)_{i, j=1 . . n}$. C'est la matrice dont la ligne $i$ est constituée par le vecteur gradient de la $i^{\text {ème }}$ composante du tenseur $T$ d'ordre 1 .

Pour un tenseur d'ordre $2, \vec{\nabla} T$ admet la représentation "matricielle" suivante : $\left(\frac{\partial T_{i j}}{\partial x_{k}}\right)_{i, j, k=1 . . n}$, la généralisation aux tenseurs d'ordre supérieurs se faisant simplement.

- $\quad$ on peut également définir la divergence du tenseur, notée $\vec{\nabla} \cdot T$ qui est un tenseur d'ordre inférieur d'une unité à l'ordre du tenseur $T$; 
Pour un tenseur d'ordre 1 , la divergence est un scalaire qui vaut : $\vec{\nabla} \cdot T=\sum^{n} \frac{\partial T_{i}}{\partial x_{i}}$ $i=1$

Pour un tenseur d'ordre 2 , de représentation matricielle $T_{i j}$, la divergence est le vecteur de composantes : $\left(\sum_{j=1}^{n} \frac{\partial T_{i j}}{\partial x_{j}}\right)_{i=1 . . n}$. C'est le vecteur constitué par la divergence des vecteurs lignes de la matrice.

Dans la suite, on notera un tenseur d'une lettre soulignée d'un nombre de fois égal à l'ordre du tenseur. Ainsi le tenseur $T$ d'ordre 4 sera écrit : $\underline{\underline{\underline{\underline{T}}}}$.

\section{b. Moment tensoriel d'ordre 2}

A partir de ces considérations, on peut envisager d'étendre la notion scalaire d'énergie à une notion tensorielle de l'énergie. Pour cela on définit donc le moment tensoriel d'ordre 2, $\underline{\underline{E}}(\vec{r}, t)$ qui est associé au "polynôme" $\boldsymbol{m} \overrightarrow{\boldsymbol{v}} \otimes \overrightarrow{\boldsymbol{v}}$ :

$\underline{\underline{\underline{E}}}(\vec{r}, t)=\iiint_{\boldsymbol{m}} \overrightarrow{\boldsymbol{v}} \otimes \overrightarrow{\boldsymbol{v}} f(\vec{r}, \vec{v}, t) d^{3} \vec{v}$ AI-43

$\underline{\underline{E}}(\vec{r}, t)$ est le tenseur énergie totale de la population, et contient donc une partie énergie cinétique et une partie énergie thermique. En reprenant les calculs précédents, et en remplaçant le produit scalaire par le produit tensoriel, nous pouvons récrire $\underline{\underline{E}}(\vec{r}, t)$ sous la forme :

$\underline{\underline{E}}=n m \vec{u} \otimes \vec{u}+m \iiint_{\vec{c}} \otimes \vec{c} f_{c}(\vec{r}, \vec{c}, t) d^{3} \vec{c}$

La première partie concerne l'énergie cinétique de translation et la seconde la partie

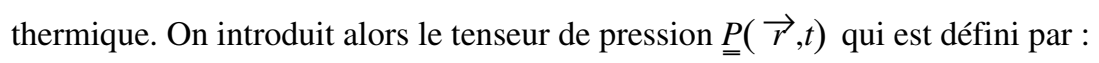

$\underline{\underline{P}}(\vec{r}, t)=m \iiint_{\vec{c}} \otimes \vec{c} f_{c}(\vec{r}, \vec{c}, t) d^{3} \vec{c}$

Ainsi que nous l'avons défini, la représentation du produit tensoriel $\vec{c} \otimes \vec{c}$ dans une base est une matrice. Si nous décomposons le vecteur $\vec{c}$ sur une base canonique, dans laquelle ses composantes sont $\left\{c_{i}\right\}_{i=1.3}$, le produit tensoriel $\vec{c} \otimes \vec{c}$ sera représenté dans cette base par la matrice de composantes $\left(c_{i} c_{j}\right)_{i, j=1.3}$, et donc le tenseur $\underset{\underline{P}}{(}(\vec{r}, t)$ par la matrice de composantes $\left(P_{i j}\right)_{i, j=1 . .3}$ définies par :

$P_{i j}=m \iiint_{c_{i}} c_{j} f_{c}(\vec{r}, \vec{c}, t) d^{3} \vec{c}$ AI-46 
Nous constatons que $\underline{\underline{P}}$ est symétrique et est donc entièrement déterminé par la donnée de 6 paramètres. $\underline{\underline{P}}$ est tel que l'on a :

$$
\begin{aligned}
\operatorname{tr}(\underline{\underline{P}}) & =m \iiint_{\left(c_{1}{ }^{2}+c_{2}{ }^{2}+c_{3}{ }^{2}\right) f_{c}(\vec{r}, \vec{c}, t) d^{3} \vec{c}} \\
& =m \iiint_{c^{2} f_{c}(\vec{r}, \vec{c}, t) d^{3} \vec{c}}
\end{aligned}
$$

Si l'on considère les trois directions équiprobables, et donc que le tenseur de pression est isotrope, la trace de ce tenseur doit vérifier :

$\operatorname{tr}(\underline{\underline{P}})=3 p$

où le facteur 3 provient des trois directions considérées et $p$ est la pression scalaire. Par ailleurs, on peut alors écrire à partir de $A I-47$ :

$\varepsilon_{t h}=\frac{1}{2} \operatorname{tr}(\underline{\underline{P}})$

et on trouve alors que la pression scalaire $p$ est la pression cinétique définie précédemment. $\underline{\underline{P}}$ contient donc toutes les informations scalaires introduites au paragraphe précédent. Nous nous sommes concentrés sur la partie diagonale, mais on peut développer le tenseur de pression sous la forme suivante :

$\underline{\underline{P}}=\frac{1}{3} \operatorname{tr}(\underline{\underline{P}}) \underline{\underline{I}}+\underline{\underline{\tau}}=p \underline{\underline{I}}+\underline{\underline{\tau}}$

où $\underline{\underline{I}}$ est le tenseur identité (qui vérifie $\operatorname{tr}(\underline{\underline{I}})=3$ ), et $\underline{\underline{\tau}}$ un tenseur que l'on appelle tenseur des contraintes. Par construction, ce dernier est un tenseur symétrique de trace nulle. En écrivant $\underline{\underline{P}}$ sous cette forme, on met en évidence la partie qui contribue à définir l'énergie du système (terme $p \underline{\underline{I}}$ ).

La partie diagonale traduit des échanges d'énergie au sein du gaz (distribution de l'énergie entre les différentes directions) et caractérise l'anisotropie du milieu. Cette anisotropie apparaît lorsqu'une direction se trouve privilégiée par rapport aux autres et que les mécanismes physiques se développent différemment suivant cette direction. C'est le cas de l'ionosphère aurorale terrestre, où le champ magnétique organise le mouvement des particules chargées en contraignant le déplacement de ces particules perpendiculairement aux lignes de champ, sans influer (en première approximation) sur le mouvement le long de la ligne de champ.

Pour quantifier cette anisotropie, on sera amené à introduire une pression et une température suivant chaque direction par :

$p_{i}=n k_{b} T_{i}=m \iiint c_{i}^{2} f_{c}(\vec{r}, \vec{c}, t) d^{3} \vec{c}$ 
Nous définirons alors une pression $p$ et une température $T$ moyennes de la manière suivante :

$$
\left\{\begin{array}{l}
p=\frac{1}{3} \sum_{i=1}^{3} p_{i} \\
T=\frac{1}{3} \sum_{i=1}^{3} T_{i}
\end{array}\right.
$$

Si une direction (notée //) est privilégiée par rapport aux deux autres (notées $\perp$ ), pour lesquelles il y a isotropie, on écrira :

$$
\left\{\begin{array}{l}
p=\frac{p_{/ /}+2 p_{\perp}}{3} \\
T=\frac{T_{/ /}+2 T_{\perp}}{3}
\end{array}\right.
$$

\section{MOMENT VECTORIEL D'ORDRE 3 : FLUX DE CHALEUR}

Si la fonction de distribution $f_{c}$ est symétrique (comme c'est le cas de la fonction de distribution de Maxwell), il y a autant d'énergie transportée dans une direction que dans la direction opposée, et le flux net est nul. Par contre, si une dissymétrie existe, un flux d'énergie apparaît et un transport d'énergie s'effectue dans la direction de la dissymétrie. On quantifie ce flux, appelé flux de chaleur, par le moment vectoriel d'ordre 3 associé au polynôme $\frac{1}{2} m c^{2} \vec{c}$ :

$$
\vec{q}(\vec{r}, t)=\iiint \frac{1}{2} \boldsymbol{m} \boldsymbol{c}^{2} \overrightarrow{\boldsymbol{c}} f_{c}(\vec{r}, \vec{c}, t) d^{3} \vec{c}
$$

En général, les dissymétries naissent d'interactions entre particules de volumes différents.

En effet, considérons deux populations de particules identiques, placés dans deux volumes infiniment grands adjacents mais séparés, en équilibre thermodynamique avec des températures différentes (chaque population est caractérisée par une fonction de distribution de Maxwell). Lorsque l'on met en communication les deux volumes, les fonctions de distributions à l'interface font apparaître une dissymétrie liée aux équilibres thermodynamiques différents. Dans chacun des réservoirs, les particules du réservoir de droite s'écoulant vers celui de gauche n'ont pas la même température que les particules s'écoulant du réservoir de gauche vers celui de droite, et leur contribution à la fonction de distribution à l'interface fait apparaître des dissymétries dans la direction de l'écoulement. Parallèlement, le mélange des deux populations a pour effet de transférer de l'énergie de la partie chaude vers la partie froide, dans la même direction que le flux associé à la dissymétrie de la fonction de distribution à l'interface. Les collisions assurent par la suite un retour à l'équilibre et à la symétrie de la distribution. 
Le flux de chaleur est le vecteur de transport de l'énergie thermique des régions "chaudes" vers les régions "froides". Toutefois, cette approche est à nuancer dans le cas des gaz multiespèces, où des phénomènes de diffusion moléculaire interviennent.

\section{EQUATION CINÉTIQUE DE TRANSPORT : ÉQUATION DE BOLTZMANN}

$\mathrm{Si}$, dans un premier temps nous excluons tous les processus à caractère inélastique (comme les réactions chimiques), et nous ne considérons que des forces conservatives, l'évolution du système caractérisé par sa fonction de distribution $f_{p}(\vec{r}, \vec{p}, t)$ est telle que le nombre de particule au sein d'un élément de volume de l'espace des phases est conservé au cours du temps, mais se déplace dans cet espace, le long d'une trajectoire qui est la trace de cette évolution.

Cette propriété se traduit mathématiquement par :

$\frac{d}{d t}\left[f_{p}(\vec{r}, \vec{p}, t) d^{3} \vec{r} d^{3} \vec{p}\right]=0$

où $\frac{d}{d t}$ représente la dérivée totale, c'est-à-dire, la dérivée par rapport au temps considérant que le volume se déplace au cours du temps dans l'espace. Or pour des forces conservatives, le volume élémentaire dans l'espace des phases reste constant et nous pouvons donc récrire $A I-55$ :

$\frac{d}{d t}\left(f_{p}(\vec{r}, \vec{p}, t)\right)=0$

Le fait que le système décrive une trajectoire dans l'espace des phases signifie, de manière implicite, que la position $\vec{r}$ et l'impulsion $\vec{p}$ sont des fonctions du temps le long de la trajectoire. L'équation $A I-56$ devient alors :

$\frac{d}{d t}\left(f_{p}(\vec{r}, \vec{p}, t)\right)=\frac{\partial f_{p}}{\partial t}+\frac{d \vec{r}}{d t} \vec{\nabla}_{r} f_{p}+\frac{d \vec{p}}{d t} \vec{\nabla}_{p} f_{p}=0$

où $\vec{\nabla}_{r}\left(f_{p}\right)$ exprime le gradient de la fonction de distribution par rapport aux directions de position $\vec{r}$ et $\vec{\nabla}_{p}\left(f_{p}\right)$ le gradient par rapport aux direction d'impulsion $\vec{p}$. Par définition la vitesse $\vec{v}$ vaut $\vec{v}=\frac{d \vec{r}}{d t}$ et le principe fondamental donne $\frac{d \vec{p}}{d t}=\vec{F}$, où $\vec{F}$ est la somme des forces extérieures agissant sur une particule du gaz. On en déduit donc l'équation suivante :

$\frac{\partial f_{p}}{\partial t}+\vec{v} \cdot \vec{\nabla}_{r} f_{p}+\vec{F} \cdot \vec{\nabla}_{p} f_{p}=0$ AI-58 
L'équation $A I-57$ (ou son équivalent $A I-58$ ) est valable, même si les particules sont relativistes. Dans le cas où elles ne le sont pas et où le champ électromagnétique qui éventuellement agit sur les particules est faible, nous avons comme relation entre la vitesse et l'impulsion : $\vec{p}=m \vec{v}$ et on peut donc transcrire l'équation AI-58 en terme d'équation d'évolution de la fonction de distribution $f(\vec{r}, \vec{v}, t)$, qui caractérise la population dans une représentation position-vitesse :

$\frac{\partial f}{\partial t}+\vec{v} \cdot \vec{\nabla}_{r} f+\frac{\vec{F}}{m} \cdot \vec{\nabla}_{v} f=0$

Cette équation peut également être écrite en terme de flux. En effet, comme les forces sont conservatives, nous avons : $\frac{\vec{F}}{m} \vec{\nabla}_{v} f=\vec{\nabla}_{v} \cdot\left(\frac{\vec{F}}{m} f\right)$ et donc :

$\frac{\partial f}{\partial t}+\vec{\nabla}_{r} \cdot(\vec{v} f)+\vec{\nabla}_{v} \cdot\left(\frac{\vec{F}}{m} f\right)=0$

Cette forme, qui est strictement identique à la forme $A I-59$ présente l'intérêt de pouvoir être généralisée à des forces non conservatives, ce que nous admettrons ici. Dans l'absolu, toutes les forces que nous considérons sont conservatives. Toutefois, leur expression n'est pas toujours facile à établir, surtout si elles mettent en jeu des phénomènes microscopiques du type collisions binaires, et dans la plupart des cas, nous n'avons accès qu'à une représentation macroscopique approchée qui nous fournit une forme non conservative de l'interaction. Par ailleurs, les interactions binaires peuvent être modélisées assez finement et n'aboutir à aucune forme analytique simple, mais à un opérateur agissant sur la fonction de distribution : l'opérateur de collisions. Nous séparerons donc les forces regroupées dans le dénominatif $\vec{F}$ en deux groupes :

- d'une part, les forces pour lesquelles nous avons une expression analytique exploitable.

Nous conserverons la dénomination $\vec{F}$ pour ces forces macroscopiques.

- d'autre part, les forces microscopiques $\vec{F}_{\text {micro }}$ pour lesquelles nous n'avons pas d'expression simple et qui s'expriment comme des fonctions de $f$. Pour ces forces, nous poserons : $\frac{1}{m} \vec{\nabla}_{v} \cdot\left(\vec{F}_{\text {micro }} f\right)=-\left.\frac{\delta f}{\delta t}\right|_{\text {coll }}$ où $\left.\frac{\delta f}{\delta t}\right|_{\text {coll }}$ est un opérateur agissant sur la fonction de distribution $f$ que l'on appelle opérateur de collisions. En introduisant cet opérateur, on peut alors généraliser l'équation aux processus chimiques, moyennant l'introduction d'un opérateur de chimie en lieu et place de l'opérateur de collisions. Dans la suite, nous supposerons donc que l'opérateur de collision recouvre toutes les formes de collisions (élastiques, inélastiques, réactives ...).

L'équation $A I-60$ prend alors la forme suivante :

$\frac{\partial f}{\partial t}+\vec{\nabla}_{r} \cdot(\vec{v} f)+\vec{\nabla}_{v} \cdot\left(\frac{\vec{F}}{m} f\right)=\left.\frac{\delta f}{\delta t}\right|_{\text {coll }}$ 
et dans la limite où les forces $\vec{F}$ sont conservatives :

$\frac{\partial f}{\partial t}+\vec{v} \cdot \vec{\nabla}_{r} f+\frac{\vec{F}}{m} \cdot \vec{\nabla}_{v} f=\left.\frac{\delta f}{\delta t}\right|_{c o l l}$

L'équation AI-62 porte le nom d'équation de Boltzmann. C'est l'équation que Boltzmann a établie en 1872 pour étudier l'évolution d'un gaz hors équilibre. Le terme $\vec{v} \cdot \vec{\nabla}_{r} f$ est parfois appelé le terme de flux libre et correspond en fait à un terme de diffusion thermique. Sa dépendance explicite vis à vis de la vitesse a une conséquence que nous verrons pour l'établissement des systèmes d'équations de transport "fluide". Il entraîne un couplage entre les différents moments de la fonction de distribution tel qu'il en résulte un problème difficile de fermeture de ces systèmes

\section{LE PASSAGE AU MACROSCOPIQUE : LES MOMENTS DE L'ÉQUATION DE BOLTZMANN}

Dans l'équation de Boltzmann que nous venons d'établir, nous avons éludé la complexité liée à l'établissement de l'opérateur de collisions. Dans la réalité, pour chaque interaction binaire (voir la description en début de paragraphe), nous devons introduire un opérateur spécifique ; nous avons donné l'exemple d'un tel opérateur pour les processus chimiques au chapitre $I V$. La conséquence de cela est une difficulté de mise en œuvre de l'équation aux altitudes ionosphériques où les mécanismes collisionels sont très importants. Il s'avère alors que la résolution directe de l'équation de Boltzmann est inappropriée et on préfère donc adopter une approche régressive où on cherche à résoudre l'évolution des paramètres macroscopiques caractérisant le système que nous avons introduits. Cette nouvelle approche correspond à l'approche "fluide" et aboutit aux équations magnétohydrodynamiqes de transport. Dans la suite, nous allons donc établir ces équations pour une population $s$ et par conséquent tous les paramètres macroscopiques seront écrits avec l'indice $s$.

Pour établir l'équation régissant l'évolution au cours du temps d'un moment de la fonction de distribution $f_{s}(\vec{r}, \vec{v}, t)$ de l'espèce $s$ la procédure est relativement simple : il suffit de multiplier l'équation de Boltzmann AI-62 par le polynôme de la vitesse qui définit le moment puis d'intégrer le résultat sur les dimensions des vitesses. Le résultat est une équation d'évolution dont les coefficients dépendent des moments de la fonction de distribution. Nous ne développerons pas plus la méthode et dans la suite nous donnerons les équations auxquelles on parvient, ainsi que les systèmes d'équations que l'on peut définir. En particulier, nous mettrons en exergue la hiérarchisation de ces équations et les techniques utilisées pour procéder à la fermeture de ces systèmes. 


\section{EQUATION DE CONTINUITÉ}

Cette équation s'obtient en multipliant par 1 et en intégrant le résultat ; ceci correspond à une intégration directe de l'équation de Boltzmann. Nous allons décrire la procédure pour cette équation ; l'application aux autres équations se faisant de manière semblable.

L'équation de Boltzmann contient quatre termes à intégrer que nous allons traiter successivement

a. Intégration de la dérivée temporelle $\frac{\partial f_{s}}{\partial t}$

Cette intégration est directe :

$\iiint_{1} 1 \frac{\partial f_{s}}{\partial t} d^{3} \vec{v}=\frac{\partial}{\partial t}\left(\iiint_{f_{s}} d^{3} \vec{v}\right)=\frac{\partial n_{s}}{\partial t}$

car l'opérateur "dérivée par rapport au temps" commute avec l'opérateur "intégrale sur les directions des vitesses".

\section{b. Intégration du flux libre $\vec{v} \cdot \vec{\nabla}_{r} f_{s}$}

Nous avons :

$\vec{v} \cdot \vec{\nabla}_{r} f_{s}=\vec{\nabla}_{r} \cdot\left(\vec{v} f_{s}\right)$

et donc l'intégration donne :

$\iiint_{1}\left\{\vec{v} \cdot \vec{\nabla}_{r} f_{s}\right\} d^{3} \vec{v}=\vec{\nabla}_{r} \cdot\left(\iiint \vec{v} f_{s} d^{3} \vec{v}\right)=\vec{\nabla}_{r} \cdot\left(n_{s} \vec{u}\right)$

car l'opérateur "divergence par rapport aux directions d'espace" commute avec l'opérateur "intégrale sur les directions des vitesses".

c. Intégration du terme $\vec{\nabla}_{v} \cdot\left(\frac{\vec{F}}{m_{s}} f_{s}\right)$

D'après le théorème de Green-Ostrogradsky, l'intégration de la divergence d'une grandeur sur un volume fermé est égale au flux de cette grandeur sur la surface délimitant ce volume. Ici le volume n'est pas fermé car nous intégrons sur tout le sous-espace des vitesses. Par contre, nous savons que la fonction de distribution $f_{s}(\vec{r}, \vec{v}, t)$ tend vers $O$ lorsque le module de la vitesse tend vers l'infini, de telle manière que le flux d'une grandeur associée à $f_{s}$ sur un volume fermé quelconque, qui inclut une sphère de rayon tendant vers l'infini, tend également vers 0 . La conséquence en est que la limite, qui correspond alors à l'intégrale sur le sous-espace tout entier, est nulle. On peut donc écrire :

$\iiint_{1}\left\{\vec{\nabla}_{v} \cdot\left(\frac{\vec{F}}{m_{s}} f_{s}\right)\right\} d^{3} \vec{v}=\overrightarrow{0}$ 
Dans la suite, nous ne considérerons que les forces électromagnétiques et la force de gravité qui sont les principales contributions aux efforts exercées sur les particules du plasma.

d. Intégration de l'opérateur de collision $\left.\frac{\delta f}{\delta t}\right|_{\text {coll }}$

Les interactions binaires conservent l'intégrité des particules qui entrent en collisions et par conséquent, leur contribution à l'équation de continuité est nulle : elles ne modifient pas le nombre de particules de l'espèce $s$. Il en est de même pour les interactions binaires inélastiques qui n'affectent pas la nature des particules mais ne conservent pas l'énergie (par exemple transfert d'énergie cinétique en état d'excitation d'une molécule puis désexcitation par rayonnement). Seules les collisions réactives, décrites au chapitre $I V$ donnent une contribution non nulle car elles affectent le nombre de particules. Pour les opérateurs associés à ces collisions, l'intégrale donne le taux de réaction (voir chapitre $I V$ ). Nous dissocierons les taux correspondant à une création effective de particules de la population $s$, que nous dénommerons $P_{s}$, des taux correspondant à une perte effective de particules de la population $s$, que nous regrouperons sous la dénomination $L_{s} n_{s}$, car le taux de réaction est alors proportionnel à $n_{s}$.

Nous pouvons donc écrire :

$\iiint_{1}\left\{\frac{\delta f}{\delta t} \mid c o l l\right\} d^{3} \vec{v}=\frac{\delta n_{s}}{\delta t}=P_{s}-L_{s} n_{s}$

\section{e. Equation de continuité}

En reprenant les développements précédents, nous parvenons à l'équation de continuité suivante :

$\frac{\partial n_{s}}{\partial t}+\vec{\nabla} \cdot\left(n_{s} \overrightarrow{u_{s}}\right)=\frac{\delta n_{s}}{\delta t}=P_{s}-L_{s} n_{s}$

Cette équation traduit la conservation de la matière lorsque le terme $\frac{\delta n_{s}}{\delta t}$ est nul. Lorsque ce terme est non nul, cette équation caractérise la dynamique chimique dans un milieu non fermé qui peut être en mouvement.

\section{EQUATION DE TRANSPORT DE LA QUANTITÉ DE MOUVEMENT (OU IMPULSION) ET DE LA VITESSE}

C'est l'équation qui s'obtient en considérant le polynôme $\boldsymbol{m}_{\boldsymbol{s}} \overrightarrow{\boldsymbol{v}}$. Le résultat prend la forme suivante :

$\frac{\partial}{\partial t}\left(n_{s} m_{s} \overrightarrow{u_{s}}\right)+\vec{\nabla}\left(n_{s} m_{s} \overrightarrow{u_{s}} \otimes \overrightarrow{u_{s}}\right)+\vec{\nabla} \cdot \underline{\underline{P}}{ }_{s}-n_{s} m_{s} \vec{g}-n_{s} e_{s}\left(\vec{E}+\overrightarrow{u_{s}} \wedge \vec{B}\right)=\frac{\delta \vec{M}_{s}}{\delta t} \quad A I-69$ 
où $\frac{\delta \vec{M}_{s}}{\delta t}$ représente la contribution des opérateurs de collisions à l'équation de transport et en pratique, la contribution principale est celle des interactions élastiques qui ont pour effet de modifier la quantité de mouvement et l'énergie des particules. Cette équation est la généralisation du principe fondamental de la dynamique.

Parfois, cette équation s'avère inadaptée à un traitement numérique et on réfère établir l'équation de transport de la vitesse $\overrightarrow{u_{s}}$. Cette dernière s'obtient en écrivant l'équation de Boltzmann dans le repère local pour la fonction de distribution $f_{c}(\vec{r}, \vec{c}, t)$ que nous avons déjà introduite. Nous n'établirons pas l'équation pour $f_{c}(\vec{r}, \vec{c}, t)$ car elle est relativement lourde et nous nous contenterons d'en présenter les résultats. L'équation de transport de la vitesse $\overrightarrow{u_{s}}$ prend la forme suivante :

$n_{s} m_{s} \frac{\partial \overrightarrow{u_{s}}}{\partial t}+n_{s} m_{s}\left(\overrightarrow{u_{s}} \cdot \vec{\nabla}\right) \overrightarrow{u_{s}}+\vec{\nabla} \cdot \underline{\underline{P}} s-n_{s} m_{s} \vec{g}-n_{s} e_{s}\left(\vec{E}+\overrightarrow{u_{s}} \wedge \vec{B}\right)=\frac{\delta \vec{M}_{s}}{\delta t}$

Les équations $A I-69$ et $A I-70$ ne sont pas indépendantes car leur différence correspond à l'équation $A I-68$.

Les autres moments que nous avons introduits sont associés à la fonction de distribution $f_{c}(\vec{r}, \vec{c}, t)$ et sont donc obtenus à partir de l'équation d'évolution de cette fonction de distribution dans le référentiel du centre de masse.

\section{EQUATION DE TRANSPORT DE L'ÉNERGIE}

\section{a. Energie thermique}

L'équation d'évolution de l'énergie thermique d'une population à trois degré de liberté, prend la forme suivante :

$\frac{\partial}{\partial t}\left(\frac{3}{2} p_{s}\right)+\overrightarrow{u_{s}} \cdot \vec{\nabla}\left(\frac{3}{2} p_{s}\right)+\frac{3}{2} p_{s}\left(\vec{\nabla} \cdot \overrightarrow{u_{s}}\right)+\vec{\nabla} \cdot \overrightarrow{q_{s}}+\underline{\underline{P}}_{s}: \vec{\nabla} \overrightarrow{u_{s}}=\frac{\delta E_{s}}{\delta t}$

où $\frac{3}{2} p_{s}$ est l'énergie thermique de cette population. Pour un nombre de degrés de liberté différents, il convient d'adapter l'expression de l'énergie thermique. Dans cette équation, le terme $\frac{\delta E_{s}}{\delta t}$ est associé aux transferts d'énergie dus aux processus collisionnels. Tous les types de mécanismes peuvent contribuer de manière significative; en particulier, les mécanismes réactionnels ont une chaleur de réaction qui apparaît dans cette expression. Toutefois, dans la plupart des cas, la chaleur de réaction est minime devant les transferts d'énergie par collision. 


\section{b. Tenseur de pression}

L'intégration de l'équation dans le référentiel du centre de masse donne l'équation d'évolution suivante du tenseur de pression :

$\frac{\partial \underline{\underline{P}} s}{\partial t}+\overrightarrow{u_{s}} \cdot \vec{\nabla} \underline{\underline{\underline{P}}} s+\vec{\nabla} \cdot \underline{\underline{\underline{Q}}}_{s}$

$+\underline{\underline{P}}_{s}\left(\vec{\nabla} \cdot \overrightarrow{u_{s}}\right)+\underline{\underline{P}}_{s} \cdot \vec{\nabla} \vec{u}_{s}+\left(\underline{\underline{P}}_{s} \cdot \vec{\nabla}{\overrightarrow{u_{s}}}^{T}+\frac{e_{s}}{m_{s}}\left[\vec{B} \wedge \underline{\underline{P}}_{s}-\underline{\underline{P}}_{s} \wedge \vec{B}\right]=\frac{\delta \underline{\underline{P}} s}{\delta t}\right.$

où $\frac{\delta \underline{\underline{P}}_{s}}{\delta t}$ est la contribution de l'opérateur de collision à l'équation du tenseur de pression.

Dans cette équation, nous avons introduit le tenseur de flux de chaleur $\underline{\underline{\underline{Q}}}$ (tenseur d'ordre 3 ) défini de manière générique par :

$Q_{i j k}=m \iiint_{c_{i}} c_{j} c_{k} f_{c}(\vec{r}, \vec{c}, t) d^{3} \vec{c}$

\section{EQUATION DE TRANSPORT DU FLUX DE CHALEUR}

Cette équation prend la forme suivante :

$$
\begin{aligned}
& \frac{\partial \overrightarrow{q_{s}}}{\partial t}+\left(\overrightarrow{u_{s}} \cdot \vec{\nabla}\right) \overrightarrow{q_{s}}+\left(\overrightarrow{q_{s}} \cdot \vec{\nabla}\right) \overrightarrow{u_{s}}+\overrightarrow{q_{s}}\left(\vec{\nabla} \cdot \overrightarrow{u_{s}}\right)+\underline{\underline{\underline{Q}}}_{s}: \vec{\nabla} \overrightarrow{u_{s}}+\vec{\nabla} \cdot \underline{\underline{\mu}}_{s} \\
& +\left[\frac{\partial \overrightarrow{u_{s}}}{\partial t}+\left(\overrightarrow{u_{s}} \cdot \vec{\nabla}\right) \overrightarrow{u_{s}}-\vec{g}-\frac{e_{s}}{m_{s}}\left(\vec{E}+\overrightarrow{u_{s}} \wedge \vec{B}\right)\right] \cdot\left(\underline{\underline{\tau}}+\frac{5}{2} p_{s} \underline{\underline{I}}\right) \\
& -\frac{e_{s}}{m_{s}} \overrightarrow{q_{s}} \wedge \vec{B} \\
& =\frac{\delta \overrightarrow{q_{s}}}{\delta t}-\frac{1}{n_{s} m_{s}} \frac{\delta \vec{M}_{s}}{\delta t} \cdot\left(\underline{\underline{\tau}}_{s}+\frac{5}{2} p_{s} \underline{\underline{I}}\right)
\end{aligned}
$$

où $\frac{\delta \overrightarrow{q_{s}}}{\delta t}$ est la contribution des collisions à l'équation de transport. Nous avons introduit dans cette équation le tenseur de pression d'ordre supérieur $\underline{\underline{\mu}}$ (tenseur d'ordre 2) dont l'expression générique est :

$\underline{\underline{\mu}}=\frac{1}{2} m \iiint_{c} \vec{c} \otimes \vec{c} f_{c}(\vec{r}, \vec{c}, t) d^{3} \vec{c}$ 


\section{FERMETURE DU SYSTÈME D'ÉQUATION}

On constate que quel que soit l'ordre auquel on arrête l'intégration, on introduit une dépendance vis-à-vis des moments d'ordres supérieurs. On a ainsi créé une hiérarchie d'équation qui donne une description d'autant plus fine que le nombre d'équations considérées est élevé. On a transformé la complexité de l'équation de transport de la fonction de distribution, due à l'opérateur de collision, en un système ouvert complexe d'équations couplées. Pour parvenir à une résolution du système, il faut imposer une fermeture en opérant une troncation de la hiérarchie d'équations.

Deux approches sont envisageables. L'une focalise sur l'impact de l'opérateur de collisions sur la déformation de la fonction de distribution par rapport à celle d'équilibre, et l'autre caractérise la déformation de la fonction de distribution par la nature des moments résolus.

\section{a. Fermeture "physique" : coefficients cinétiques de transport}

Cette procédure correspond à l'approche de Chapman-Enskog. Si on analyse le problème lié aux collisions, on constate que dans les régions où les collisions sont élevées, le libre parcours moyen est très petit devant les échelles caractéristiques de variation des paramètres macroscopiques. On peut donc développer la fonction de distribution à partir d'une fonction de distribution d'équilibre (typiquement une fonction de distribution de Maxwell) en utilisant comme paramètre de développement un paramètre quantifiant le rapport du libre parcours moyen aux échelles caractéristiques (nombre de Knudsen). On déduit alors des coefficients cinétiques de transport qui permettent d'exprimer les moments d'ordres supérieurs en fonction des gradients des moments d'ordres inférieurs. Ainsi :

- le coefficient de diffusion moléculaire $D$ est associé au gradient de concentration et permet d'exprimer la vitesse (loi de Fick) :

$\vec{u}=-D \frac{\vec{\nabla} n}{n}$

- le coefficient de viscosité $\eta$ est associé aux gradients de vitesse et permet d'exprimer le tenseur de contrainte :

$\underline{\underline{\tau}}=-\eta\left[\vec{\nabla} \vec{u}+(\vec{\nabla} \vec{u}) r-\frac{2}{3}(\vec{\nabla} \cdot \vec{u}) \underline{\underline{I}}\right]$

- le coefficient de conductivité thermique $\kappa$ est associé au gradient de la température et permet d'exprimer le flux de chaleur (loi de Fourier) :

$\vec{q}=-\kappa \vec{\nabla} T$

Ces coefficients sont des caractérisations macroscopiques de l'effet des collisions. De fait, leur validité n'est assurée que dans des régions où le libre parcours moyen reste faible. La troncation des équation est alors facile à faire : par exemple les équations de Navier-Stokes sont obtenues en utilisant la relation entre le tenseur des contraintes et les gradients de la vitesse. 
Nous ne nous étendrons pas sur les expressions de ces coefficients qui peuvent être obtenues à partir de l'autre méthode de fermeture ; le chapitre $V$ montre d'ailleurs comment on procède pour exprimer certains de ces coefficients.

\section{b. Fermeture mathématique}

Elle correspond à l'approche de Grad. A partir d'une fonction de distribution supposée d'équilibre (par exemple une fonction de Maxwell), on peut développer une famille ordonnée de fonctions orthogonales (au sens du produit scalaire défini en prenant la fonction d'équilibre comme fonction de pondération), sur laquelle la véritable fonction de distribution peut être développée. Les coefficients de ce développement sont alors exprimés en fonction des moments macroscopiques de la fonction de distribution recherchée. Ordonner la famille autorise d'imposer que le $p^{\text {ème }}$ coefficient du développement ne dépend que des moments d'ordre inférieur ou égal à $p$.

En conséquence, une troncation au $p^{\text {ème }}$ terme permet de déterminer le moment d'ordre $p+1$ en fonction des moments d'ordre au plus $p$, et rend possible la fermeture du système d'équations.

On peut ainsi générer différents systèmes d'équations en fonction du choix de la fonction de distribution d'équilibre et de l'ordre de la troncature.

L'hypothèse sous-jacente est que les termes d'ordres supérieurs (et donc les moments auxquels ils sont attachés) sont négligeables devant les termes d'ordres inférieurs.

Dans le cas d'une fonction de distribution d'équilibre de Maxwell, notée $f_{o}$, on peut écrire à l'ordre 3 :

$f_{c}(\vec{r}, \vec{c}, t)=f_{o}(\vec{r}, \vec{c})\left(1+\frac{m_{s}}{2 k_{b} T_{s}} \underline{\underline{\tau}}_{s}:(\vec{c} \otimes \vec{c})+\frac{m_{s}}{k_{b} T_{s} p_{s}}\left(1-\frac{m_{s} c^{2}}{5 k_{b} T_{s}}\right) \vec{q} \cdot \vec{c}\right)$

D'où on déduit :

$\underline{\underline{\underline{\underline{\mu}}}} s=\frac{5}{2} \frac{k_{b} T_{s}}{m_{s}}\left(p_{s} \underline{\underline{I}}+\frac{7}{2} \underline{\underline{\tau}} s\right)$

et :

$\left(Q_{s}\right)_{i j k}=\frac{2}{5}\left[\left(q_{s}\right)_{i} \delta_{j k}+\left(q_{s}\right)_{k} \delta_{i j}+\left(q_{s}\right)_{j} \delta_{k i}\right]$

où $\delta_{i j}$ représente le symbole de Kronecker : $\delta_{i j}=1$ si $i=j, \delta_{i j}=0$ sinon.

Nous allons décrire deux systèmes d'équations qui sont fréquemment utilisés en aéronomie en mettant en avant les différences fondamentales qui existent entre les équations.

\section{c. Système à 5 moments}

On obtient ce système en ne conservant que l'ordre 0 dans le développement $A I-79$. On considère alors que la fonction est une fonction de distribution maxwellienne, caractérisée par sa température. Le système d'équation que l'on déduit correspond aux équations d'Euler 
de la mécanique des fluides lorsqu'on annule les termes de collisions. Ce système d'équation permet donc de résoudre l'évolution de la concentration ( 1 paramètre), de la vitesse ( 3 paramètres) et de l'énergie thermique ( 1 paramètre), d'où son nom de système à 5 moments.

La troncation implique :

$$
\left\{\begin{array}{l}
\overrightarrow{q_{s}} \quad=\overrightarrow{0} \\
\vec{\nabla} \cdot \underline{\underline{P}}_{s}=\vec{\nabla} p_{s} \\
\underline{\underline{P}}_{s}: \vec{\nabla} \vec{u}_{s}=p_{s}\left(\vec{\nabla} \cdot \overrightarrow{u_{s}}\right)
\end{array}\right.
$$

L'équation de continuité $A I-68$ reste inchangée. L'équation de transport de la vitesse devient :

$$
n_{s} m_{s} \frac{\partial \overrightarrow{u_{s}}}{\partial t}+n_{s} m_{s}\left(\overrightarrow{u_{s}} \cdot \vec{\nabla}\right) \overrightarrow{u_{s}}+\vec{\nabla} p_{s}-n_{s} m_{s} \vec{g}-n_{s} e_{s}\left(\vec{E}+\overrightarrow{u_{s}} \wedge \vec{B}\right)=\frac{\delta \overrightarrow{M_{s}}}{\delta t}
$$

et l'équation de transport de l'énergie s'écrit :

$\frac{\partial}{\partial t}\left(\frac{3}{2} p_{s}\right)+\overrightarrow{u_{s}} \cdot \vec{\nabla}\left(\frac{3}{2} p_{s}\right)+\frac{5}{2} p_{s}\left(\vec{\nabla} \cdot \overrightarrow{u_{s}}\right)=\frac{\delta E_{s}}{\delta t}$

\section{d. Système à 8 moments}

On considère le développement de la fonction de distribution jusqu'à l'ordre 3 , mais on suppose que le tenseur des contraintes $\underline{\underline{\tau}}$ est nul. Ce système d'équation permet donc de résoudre l'évolution de la concentration ( 1 paramètre), de la vitesse (3 paramètres), de l'énergie thermique ( 1 paramètre) et du flux de chaleur ( 3 paramètres), d'où son nom de système à 8 moments.

L'hypothèse dans le développement entraîne donc comme simplifications :

$$
\left\{\begin{array}{l}
\underline{\underline{\tau}}_{s} \quad=\underline{\underline{0}} \\
\vec{\nabla} \cdot \underline{\underline{\mu}}_{s}=\frac{5}{2} \frac{k_{b}}{m_{s}} \vec{\nabla}\left(T_{s} p_{s}\right) \\
\underline{\underline{\underline{Q}}}_{s}: \vec{\nabla} \vec{u}=\frac{2}{5}[\vec{q}(\vec{\nabla} \cdot \vec{u})+\vec{q} \cdot(\vec{\nabla} \vec{u})+(\vec{\nabla} \vec{u}) \cdot \vec{q}]
\end{array}\right.
$$

Les équations de continuité $A I-68$ et de transport de la vitesse $A I-83$ restent inchangées. L'équation d'énergie devient :

$\frac{\partial}{\partial t}\left(\frac{3}{2} p_{s}\right)+\overrightarrow{u_{s}} \cdot \vec{\nabla}\left(\frac{3}{2} p_{s}\right)+\frac{5}{2} p_{s}\left(\vec{\nabla} \cdot \overrightarrow{u_{s}}\right)+\vec{\nabla} \cdot \overrightarrow{q_{s}}=\frac{\delta E_{s}}{\delta t}$ AI-86 
tandis que l'équation de transport du flux de chaleur s'écrit :

$$
\begin{aligned}
& \frac{D \overrightarrow{q_{s}}}{D t}+\frac{7}{5}\left(\overrightarrow{q_{s}} \cdot \vec{\nabla}\right) \overrightarrow{u_{s}}+\frac{7}{5} \overrightarrow{q_{s}}\left(\vec{\nabla} \cdot \overrightarrow{u_{s}}\right)+\frac{2}{5}(\vec{\nabla} \vec{u}) \cdot \vec{q} \\
& +\frac{5}{2} \frac{k_{b} p_{s}}{m_{s}} \vec{\nabla} T_{s}-\frac{e_{s}}{m_{s}} \overrightarrow{q_{s}} \wedge \vec{B}=\frac{\delta \vec{q}_{s}}{\delta t}-\frac{5}{2} \frac{p_{s}}{n_{s} m_{s}} \frac{\delta \vec{M}_{s}}{\delta t}
\end{aligned}
$$

\section{EXPRESSION DES COLLISIONS}

Dans toutes les équations que nous avons présentées, nous avons mentionné les collisions sans les exprimer. Dans ce paragraphe, nous allons les écrire pour les deux systèmes que nous venons d'exposer, sans faire de description détaillée de la manière de les obtenir. Ce que nous pouvons dire, c'est que le calcul de l'intégrale de l'opérateur de collisions pour les équations de transport est une opération fastidieuse. Pour simplifier le problème, on choisit un opérateur qui représente un bon compromis entre tous les mécanismes collisionnels ; en général, on choisit l'opérateur intégral de collisions de Boltzmann.

Cependant, même avec cette simplification, le calcul de l'intégrale n'est pas aisé. En effet, les équations de transport sont obtenues en utilisant une expression approchée de la fonction de distribution, qui n'est valable que dans la limite où les termes négligées sont réellement négligeables. De plus, les fluides auxquels on a affaire, sont des fluides multiespèces où les caractéristiques de chacun des composants du fluide (température, vitesse d'écoulement, ...) sont très différentes. Pour obtenir des expressions exploitables analytiquement, la procédure qui est suivie consiste donc à développer l'opérateur de collisions avec la fonction de distribution approchée et à ne retenir que les termes d'ordre le plus bas. De fait, cette procédure limite la validité des termes de collisions aux cas où les écarts entre les paramètres macroscopiques des différentes espèces sont faibles, ce qui est généralement le cas aux altitudes ionosphériques.

\section{a. Equation de continuité}

Nous avons déjà présenté ce terme en précisant qu'il n'incluait que les contributions des processus chimiques. Nous rappelons son expression :

$\frac{\delta n_{s}}{\delta t}=P_{s}-L_{s} n_{s}$

où $P_{s}$ est le taux de production de l'espèce $s$, qui est dû soit à des mécanismes chimiques, soit à la photoionisation ou à l'ionisation par impact. Le terme $L_{s} n_{s}$ représente les pertes par réactions chimiques et le coefficient $L_{s}$ caractérise la durée de vie chimique de l'espèce $s$ vis à vis de ces réactions (voir chapitre $I V$ et annexe $I I$ ). 


\section{b. Equation de transport de la vitesse}

Suivant l'ordre auquel on faite la troncation de la fonction de distribution, le terme de collisions $\frac{\delta \vec{M}_{s}}{\delta t}$ prend différentes formes.

\section{i. Système à 5 moments}

L'expression que l'on obtient prend la forme suivante :

$\frac{\delta \vec{M}_{s}}{\delta t}=n_{s} m_{s} \sum_{t} v_{s t}\left(\overrightarrow{u_{t}}-\overrightarrow{u_{s}}\right)$

où $v_{s t}$ est la fréquence de transfert de quantité de mouvement que nous avons introduite au chapitre $I V$.

\section{ii. Système à 8 moments}

La différence essentielle avec le système à 5 moments est la prise en compte du flux de chaleur et donc de tous les effets qu'il peut avoir sur le mouvement des particules. L'expression du terme de collisions devient :

$\frac{\delta \vec{M}_{s}}{\delta t}=n_{s} m_{s} \sum\left\{v_{s t}\left(\overrightarrow{u_{t}}-\overrightarrow{u_{s}}\right)+\frac{z_{s t} \mu_{s t}}{k_{b} T_{s t}} v_{s t}\left(\frac{\overrightarrow{q_{s}}}{n_{s} m_{s}}-\frac{\overrightarrow{q_{t}}}{n_{t} m_{t}}\right)\right\}$

où $\mu_{s t}=\frac{m_{s} m_{t}}{m_{s}+m_{t}}$ est la masse réduite des espèces $s$ et $t, T_{s t}=\frac{m_{t} T_{s}+m_{s} T_{t}}{m_{s}+m_{t}}$ est la température réduite de ces mêmes espèces et $z_{s t}$ un coefficient numérique dont la valeur dépend du type d'interaction considérée. Les valeurs numériques seront données à la fin de ce chapitre.

Le premier terme est le même que dans le système à 5 moments. Le second terme caractérise les effets de diffusion thermique qui correspond à un mouvement des particules en présence d'un gradient de température. Cette expression montre l'apport essentiel du flux de chaleur dans le système à 8 moments par rapport au système à 5 moments.

\section{c. Equation de l'énergie}

Les deux systèmes admettent la même expression du terme de collision :

$\frac{\delta E_{s}}{\delta t}=\sum \frac{m_{s}}{m_{s}+m_{t}} n_{s} v_{s t}\left[3 k_{b}\left(T_{t}-T_{s}\right)+m_{t}\left|\overrightarrow{u_{t}}-\overrightarrow{u_{s}}\right|^{2}\right]$

Cette expression fait apparaître deux contributions. La nature de ces contributions est détaillée dans le chapitre $V I$ et correspondent pour la première aux échanges thermiques entre les populations et pour la seconde à la répartition entre les populations, après la collision, de l'énergie cinétique dans le référentiel du centre de masse. 


\section{d. Equation du flux de chaleur}

L'expression des collisions relative à cette équation est spécifique du système à 8 moments et s'écrit :

$$
\begin{aligned}
\frac{\delta \overrightarrow{q_{s}}}{\delta t}= & \sum v_{s t}\left(D_{s t}^{(4)} \frac{n_{s} m_{s}}{n_{t} m_{t}} \vec{q}_{t}-D_{s t}^{(1)} \overrightarrow{q_{s}}+\frac{5}{2}\left[1-\frac{m_{t} z_{s t}}{m_{s}+m_{t}}\right] p_{s}\left(\overrightarrow{u_{t}}-\overrightarrow{u_{s}}\right)\right) \\
& -\frac{2}{5} z_{s t}{ }^{\prime \prime} v_{s s} \overrightarrow{q_{e}}
\end{aligned}
$$

avec :

$$
\left\{\begin{array}{l}
D_{s t}^{(1)}=\frac{1}{\left(m_{s}+m_{t}\right)^{2}}\left(3 m_{s}^{2}-\frac{5}{2} m_{t}\left(m_{s}+m_{t}\right) z_{s t}+m_{t}^{2} z_{s t}^{\prime}+\frac{4}{5} m_{s} m_{t} z_{s t}^{\prime \prime}\right) \\
D_{s t}^{(4)}=\frac{1}{\left(m_{s}+m_{t}\right)^{2}}\left(3 m_{t}^{2}-\frac{5}{2} m_{t}\left(m_{s}+m_{t}\right) z_{s t}+m_{t}^{2} z_{s t}{ }^{\prime}-\frac{4}{5} m_{s} m_{t} z_{s t}^{\prime \prime}\right)
\end{array}\right.
$$

où $z_{s t}, z_{s t}{ }^{\prime}$ et $z_{s t}{ }^{\prime \prime}$ sont des coefficients caractérisant la nature des interactions.

Dans le terme de collisions, on voit apparaître une contribution du flux différentiel d'écoulement. Cette contribution correspond à l'effet thermodiffusif qui, dans le cas de particules chargées, est à associer à l'effet thermoélectrique. Cette contribution peut devenir prépondérante dans l'ionosphère où des courants intenses peuvent circuler. Le chapitre VI décrit plus précisément ces contributions croisées dans le cas des électrons.

\section{e. Paramètres correctifs}

Les formulations des collisions que nous avons données font apparaître des coefficients qui permettent de distinguer les types d'interaction : les expressions sont génériques et la spécificité de l'interaction est reportée d'une part dans la valeur de la fréquence de collision et d'autre part dans des facteurs de forme qui sont des caractérisations de l'interaction au travers de la loi de puissance. Nous nous contenterons de donner les valeurs numériques pour différentes loi de puissance :

- collision coulombienne : le potentiel d'interaction est en $r^{-1}$;

- collision de type polarisation (Maxwell) : le potentiel d'interaction est en $r^{-4}$;

- collision de type sphère dure : le potentiel correspond à une puissance infinie.

\begin{tabular}{|c|c|c|c|}
\cline { 2 - 4 } \multicolumn{1}{c|}{} & Coulomb & Maxwell & Sphère dure \\
\hline$z_{s t}$ & $\frac{3}{5}$ & 0 & $-\frac{1}{5}$ \\
\hline$z_{s t}{ }^{\prime}$ & $\frac{13}{10}$ & 1 & $\frac{13}{10}$ \\
\hline$z_{s t}{ }^{\prime \prime}$ & 2 & 2 & 2 \\
\hline
\end{tabular}




\section{BIBLIOGRAPHIE}

Une présentation très soignée des équations de transport peut être trouvée dans :

« Mathematical structure of transport equations for multispecies flows », par R.W. Schunk, Reviews of Geophysics and Space Physics, 429-445, 1977.

Transport processes in plasmas, R. Balescu, North Holland ed., 1988, ISBN 0-444-87093-8 (2 tomes).

Une approche différente, moins mathématique et plus "avec les mains" des plasma est donnée par :

Plasma physics and controlled fusion, F.F. Chen, Plenum Press ed., 1984, ISBN 0-30641332-9 (2 tomes).

En français, on pourra lire le très rigoureux :

Physique des plasmas, J.L. Delcroix et A. Bers, Interedition/ed. Du CNRS, 1994, ISBN 2-7296-0484-7 et 2-7296-0484-8 (2 tomes). 